CORRESPONDENCE

\title{
Venetoclax-ponatinib for T3151/compound-mutated Ph+ acute lymphoblastic leukemia
}

\author{
(c) The Author(s) 2022
}

Blood Cancer Journal (2022)12:20; https://doi.org/ 10.1038/s41408-022-00621-9

The outcome of Philadelphia chromosome-positive acute lymphoblastic leukemia $(\mathrm{Ph}+\mathrm{ALL})$ has greatly improved in the tyrosine kinase inhibitor (TKI) era and is moving to a chemo-free era using dasatinib and blinatumomab [1, 2]. However, the outcome of T315l/compound-mutated $\mathrm{Ph}+\mathrm{ALL}$ patients is dismal [3-5]. Our previous study, which included $38 \mathrm{Ph}+\mathrm{ALL}$ patients harboring the T315I mutation, showed a poor outcome, although some patients received hematopoietic stem cell transplantation (HSCT) or chimeric antigen receptor (CAR) T cell therapy [5], which represents an unmet need for new therapeutic approaches for this population. Recently, bcl-2 inhibitors have shown promising therapeutic activity in $\mathrm{Ph}+\mathrm{ALL}$ in vitro [6-8]. Lenoard et al showed that the combination of TKls and venetoclax has synergistic antileukemic efficacy in $\mathrm{Ph}+\mathrm{ALL}$ patient xenografted immunodeficient mice [7]. Moreover, Scherr et al. reported the curative potential of venetoclax-TKI-dexamethasone in a BCR-ABL + mouse model [8], thereby suggesting a novel treatment strategy for $\mathrm{Ph}+\mathrm{ALL}$. Here, we report the promising clinical results of a series of relapsed $\mathrm{Ph}+\mathrm{ALL}$ patients harboring the T315I mutation treated with venetoclax, ponatinib, and dexamethasone (VPD).

We retrospectively analyzed relapsed/refractory $(R / R) P h+A L L$ patients with $\mathrm{T} 315 \mathrm{l} / \mathrm{compound}$-mutation who were treated with venetoclax (100 mg d1, $200 \mathrm{mg} \mathrm{d} 2,400 \mathrm{mg} \mathrm{d} 3-28)$, ponatinib (45 mg d1-28) and dexamethasone $(0.15 \mathrm{mg} / \mathrm{kg} \mathrm{d} 1-21,0.075 \mathrm{mg} /$ kg d22-28) (VPD regimen) between January 2020 and May 2021 (Fig. 1A). Informed consent was obtained from all the patients. The last follow-up date is May 20, 2021. Response assessments were performed according to the NCCN guidelines for acute lymphoblastic leukemia (version 2. 2021) [9]. Event-free survival (EFS) was defined as the time from complete remission (CR) after the VPD regimen until relapse, death, or last follow-up date, whichever occurred first. Overall survival (OS) was defined as the time from the start of the VPD regimen until death or the last follow-up date, whichever occurred first. Adverse events were graded according to National Cancer Institute Common Terminology Criteria for Adverse Events, version 5.0. Bcr/abl kinase domain mutation analysis was performed by using direct Sanger sequencing. All statistical analyses were conducted using Prism software version 7.0 (GraphPad Software, La Jolla, CA, USA).

19R/R Ph+ ALL patients with the T315I/compound mutation received the VPD regimen, which included 12 males and 7 females, with a median age of 42 years (range 22 years to 74 years). Of these patients, 17 patients had de novo $\mathrm{Ph}+\mathrm{ALL}, 15 \mathrm{had}$ BCR/ABL p190, and 2 had BCR/ABL p210. Two patients had secondary $A L L$ accelerated from $C M L$, with $B C R / A B L$ p210. All patients had the $\mathrm{T} 315 \mathrm{I}$ mutation alone or compound mutation when relapsed before VPD regimen treatment, including 15 patients with the T315I mutation alone and 4 with compound kinase mutations $(100 \% \mathrm{~T} 315 \mathrm{I}+50 \%$ E255K/V, 100\% T315I+ $100 \%$ E279K, $100 \%$ T315I + 100\% Y $253 \mathrm{H}$, and $54 \%$ G250E $+36 \%$ F359V for each). BCR-ABL1 compound mutants were defined as harboring $\geq 2$ mutations in the same BCR-ABL1 allele [10]. The first three patients were considered as compound mutation because of their individual $100 \%$ T315I mutant although we just performed Sanger sequencing but not clonal sequencing. While the patient with $54 \%$ G250E and 36\% F359V mutant was not verified by clonal sequencing because of running out of sample.19 patients had previously received a median of 3 lines of salvage therapy (range: 1-6), including all patients who failed TKI (imatinib, dasatinib, nilotinib, flumatinib, or ponatinib) with or without chemotherapy, 5 patients who failed CAR T cell therapy, 1 who failed venetoclax, and 1 who failed allogeneic hematopoietic stem cell transplantation (allo-HSCT) (Table 1, Table S1).

After one cycle, 17 patients (89.5\%) achieved CR/CRi [14 minimal residual diseases (MRD) - negative by flow cytometry; 11 major molecular remission (MMR); 8 complete molecular remissions (CMR)] (Fig. 1B, Table 1, Table S1). For post-remission treatment, 6 patients who attained CMR directly received allo-HSCT, and the remaining 11 patients continued to receive the VPD regimen. Four of 11 patients attained CMR later during VPD consolidation treatment. The other two patients who did not respond did not achieve CR/CRi later. For 6 patients who received allo-HSCT, the type of donor, intensity of conditioning regimen, and timing of BMT are listed in Table S2. Five patients received haploidentical transplantation, and the other patient received $9 / 10$ unrelated donor transplantation. All 6 patients received a modified busulfan $(\mathrm{BU})$ and cyclophosphamide $(\mathrm{CY})$ conditioning regimen prior to allo-HSCT when they attained CMR after one cycle of the VPD regimen. Subsequently, relapse occurred in 1/6 [allo-HSCT group)] and $7 / 11$ (VPD consolidation group). Of the 6 patients who received allo-HSCT, 2/6 (patient 1 and patient 19) received $45 \mathrm{mg}$ of ponatinib per day as maintenance treatment, and 4 other patients did not receive maintenance treatment. At a median follow-up of 259 days, the median EFS and OS of patients starting VPD treatment were 242 and 400 days, respectively.

Adverse events of the VPD regimen were listed in Fig. 1C. Grade 3-4 neutropenia, anemia, and thrombocytopenia occurred in $73.7 \%, 36.8 \%$, and $52.6 \%$ of patients, respectively. A total of $5.3 \%$ and $16 \%$ of patients had grade $3-4$ rash and infection, respectively. Patient 12 with grade 3 rash and patient 15 with grade 2 rash reduced the ponatinib dose from $45 \mathrm{mg}$ per day to $30 \mathrm{mg}$ per day. No tumor lysis syndrome or death occurred. 7/19 patients were treated safely outpatient.

Moreover, venetoclax had a strong synergistic effect with ponatinib and dexamethasone on inducing apoptosis of primary blast cells and BaF3 cells expressing p190 BCR/ABL with the T315I mutation in vitro, with a combination index of 0.019 when the suppression rate was 0.05 , while the effect was significantly 


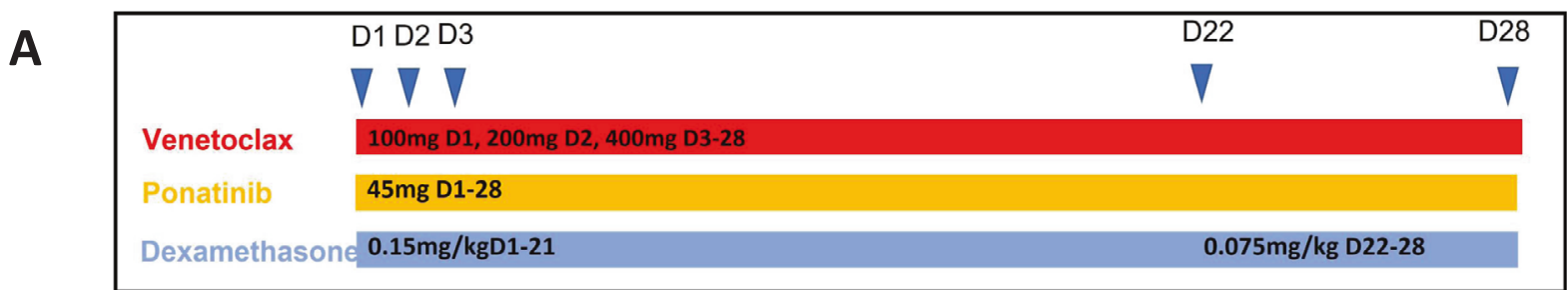

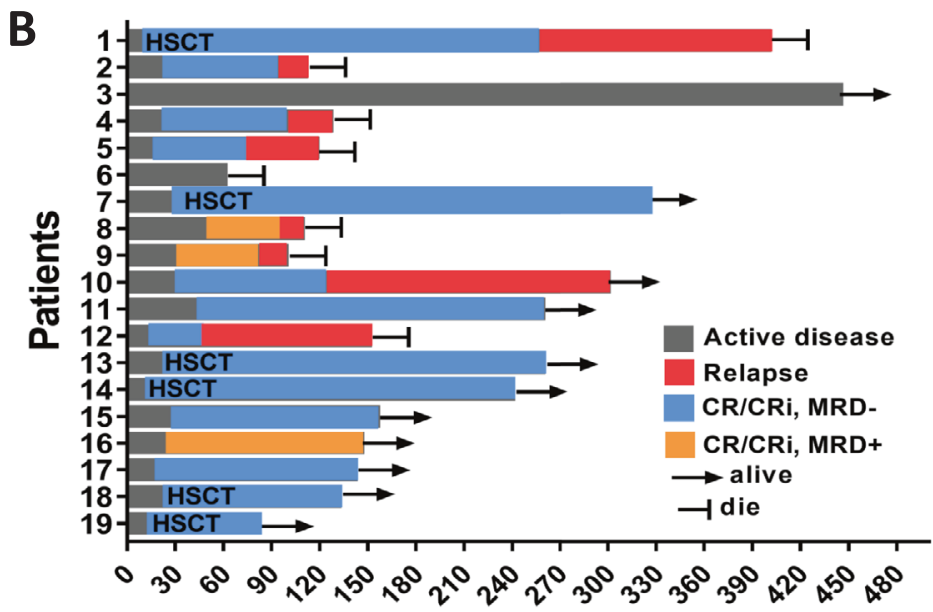

\section{Days}

C
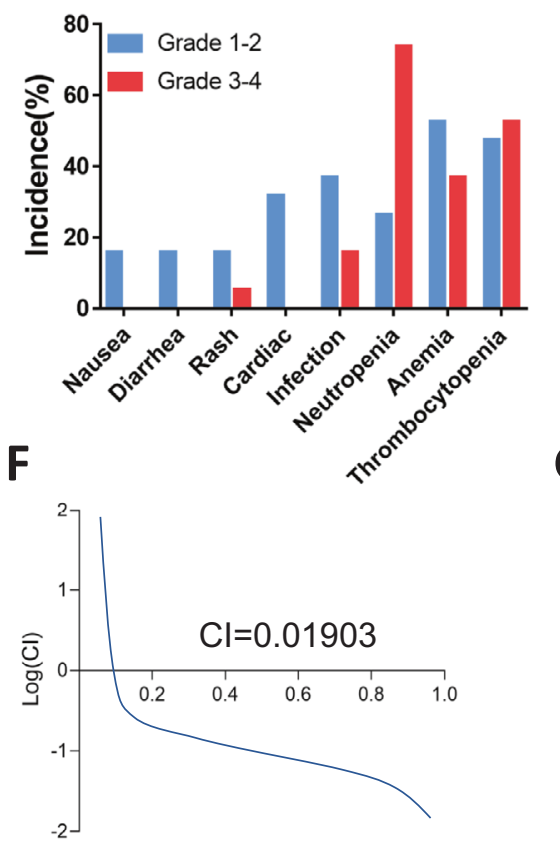

D

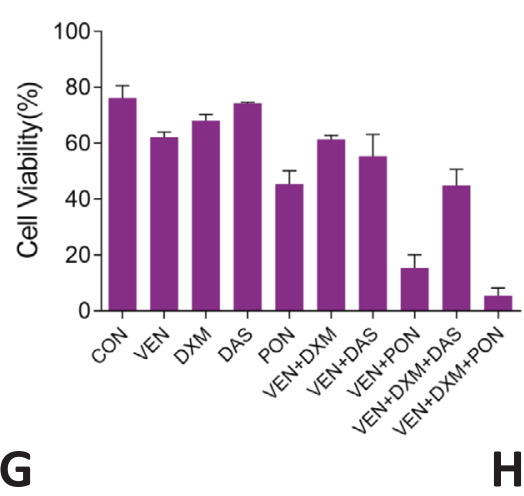

G

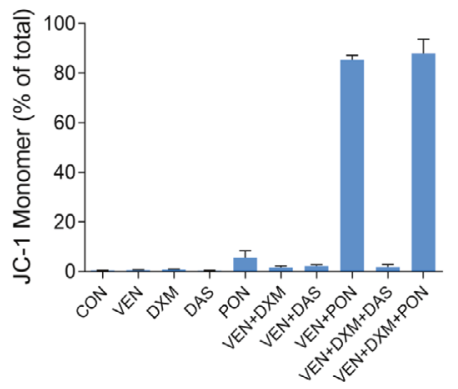

E
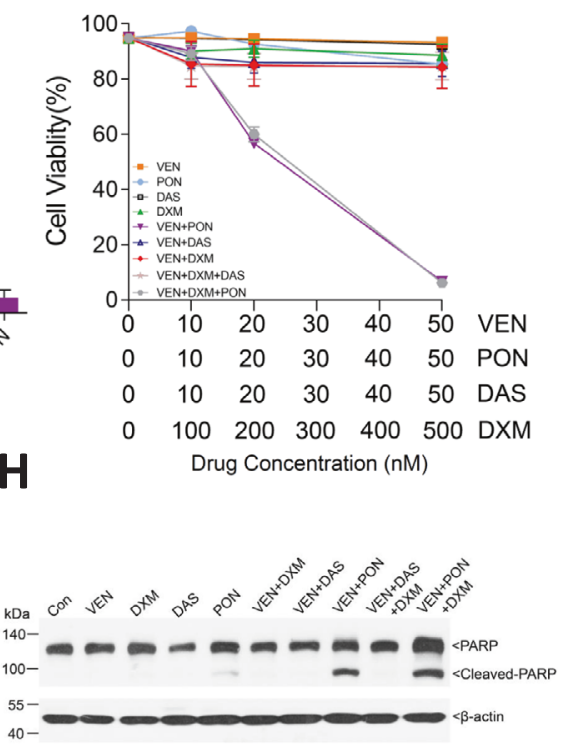

Fig. 1 Clinical response and In vitro results of the VPD regimen. A Trial scheme. B Shown is the clinical outcome for the 19 refractory/ relapsed $\mathrm{Ph}+\mathrm{ALL}$ patients with T315I or compound mutation who were treated with a VPD regimen. Response assessments were performed according to the NCCN guidelines Acute Lymphoblastic Leukemia (version 2. 2021). The indicated responses include a complete response (CR) and CR with incomplete count recovery (CRi). C Adverse Events of VPD regimen. D Mononuclear cells separated from one Ph+ ALL patient harboring T315I mutation were treated with venetoclax (VEN), ponatinib (PON), dasatinib (DAS), dexamethasone (DXM) alone or in combination at the indicated concentrations for $24 \mathrm{~h}$. Apoptosis, as indicated by flow cytometry after Annexin V/PI double staining. E-H BaF3 cells expressing p190 BCR-ABL T315I mutation were treated with VEN, PON, DAS, DXM alone or in combination at the indicated concentrations for $24 \mathrm{~h}$. Apoptosis, as indicated by flow cytometry after Annexin V/PI double staining (E), combination index (Cl) of three drugs by using CompuSyn software (F), mitochondrial were stained with JC-1 (G), and western-blot analysis of PARP and cleaved-PARP expression (H). Notes: MRD minimal residual disease, as assessed by flow cytometry. HSCT hematopoietic stem-cell transplantation. 
Table 1. Baseline characteristics of $\mathrm{Ph}+\mathrm{ALL}$ patients.

\begin{tabular}{|c|c|}
\hline Characteristics & All $\mathrm{r}$ \\
\hline \multicolumn{2}{|l|}{ Age, years } \\
\hline Median & 42 \\
\hline Range & $22-74$ \\
\hline \multicolumn{2}{|l|}{ Sex, $n(\%)$} \\
\hline Male & $12(63.2)$ \\
\hline Female & $7(36.8)$ \\
\hline \multicolumn{2}{|l|}{$B C R / A B L, n(\%)$} \\
\hline P190 & $15(78.9)$ \\
\hline P210 & $4(21.1)$ \\
\hline \multicolumn{2}{|l|}{ ABL kinase domain, $n$ (\%) } \\
\hline T315I alone & $15(78.9)$ \\
\hline T315I and E255K/V & $1(5.3)$ \\
\hline T315I and E279K & $1(5.3)$ \\
\hline $\mathrm{T} 315 \mathrm{I}$ and $\mathrm{Y} 253 \mathrm{H}$ & $1(5.3)$ \\
\hline G250E and F359V & $1(5.3)$ \\
\hline Previous lines of therapy, median(range) & $3(1-6)$ \\
\hline Failure to $\mathrm{TKI} \pm$ chemotherapy, $n(\%)$ & $19(100)$ \\
\hline Failure to venetoclax, $n(\%)$ & $1(5.3)$ \\
\hline Failure to CAR-T therapy, $n(\%)$ & $5(26.3)$ \\
\hline Failure to allo-HSCT, $n$ (\%) & $1(5.3)$ \\
\hline \multicolumn{2}{|l|}{ Response after one cycle of VPD, $n$ (\%) } \\
\hline $\mathrm{CR}$ & $13(68.4)$ \\
\hline $\mathrm{CRi}$ & $4(21.1)$ \\
\hline NR & $2(10.5)$ \\
\hline \multicolumn{2}{|l|}{ MRD-FCM in responder, $n$ (\%) } \\
\hline Negative & $14(82.4)$ \\
\hline Positive & $3(17.6)$ \\
\hline MMR in responder, $n(\%)$ & $11(64.7)$ \\
\hline CMR in responder, $n$ (\%) & $8(47.1)$ \\
\hline TOR in responder, median (range) & $25(10-53)$ \\
\hline \multicolumn{2}{|l|}{ Post-remission, $n$ (\%) } \\
\hline Allo-HSCT group & $6(35.3)$ \\
\hline VPD group & $11(64.7)$ \\
\hline Relapse, $n(\%)$ & $8(47.1)$ \\
\hline Allo-HSCT group & $1(16.7)$ \\
\hline VPD group & 7 (63.6) \\
\hline
\end{tabular}

TKIs tyrosine kinase inhibitors, CAR-T chimeric antigen receptor-T, Allo-HSCT allogeneic hematopoietic cell transplantation, $C R$ complete remission, $C R i$ $C R$ with incomplete count recovery, $N R$ no remission, MRD-FCM minimal residual disease detected by flow cytometry, TOR time to response, TOMMR time to major molecular remission. ${ }^{*}$ From the starting day of the VPD regimen.

decreased when ponatinib was replaced by dasatinib (Fig. 1D-F). A prominent change in mitochondrial membrane potential and cleavage of PARP was also observed in the triple-combination treatment group (Fig. 1G, H). Unfortunately, only one patient sample was available, and more patient samples need to be verified in the future.

For $\mathrm{T} 315 \mathrm{l} /$ compound-mutated $\mathrm{Ph}+\mathrm{ALL}$, the VPD regimen exhibited an $89.5 \% \mathrm{CR} / \mathrm{CRi}$ rate, with deep molecular remission (57.9\% MMR), while ponatinib alone showed a $41 \%$ hematologic response [3], which was supported by preclinical data suggesting that TKIs and venetoclax are highly synergistic in BCR-ABL + cells in vitro. $A$ total of $7 / 11$ and $1 / 6$ patients subsequently relapsed in the continuous VPD and allo-HSCT post-remission treatment groups, respectively, suggesting that bridging to allo-HSCT after remission is warranted. Moreover, novel compounds such as blinatumomab showed a preliminary efficacy [11]. Comparisons of efficacy in different salvage regimens for R/R Ph+ ALL with T315I or compound mutations are listed in Table S3. In summary, the VPD regimen provides a novel treatment for T315I/compoundmutated $\mathrm{R} / \mathrm{R} \mathrm{Ph}+\mathrm{ALL}$ under a complete oral and chemo-free model. A clinical trial also using a similar VPD regimen for the treatment of $R / R P h+A L L$ is ongoing now, and the results of a phase 1 portion of this study including nine patients also showed that the combination of ponatinib, venetoclax, and dexamethasone in $R / R P h+A L L$ is safe and effective [12].

Huafeng Wang ${ }^{1,2,3,4,12}$, Chang Yang ${ }^{1,5,12}$, Ting Shi ${ }^{1,2,3,12}$ Yi Zhang ${ }^{1,2,3,12}$, Jiejing Qian ${ }^{1,2,3,12}$, Yúngui Wang ${ }^{1,2,3^{\prime}}$, Yongxian $\mathrm{Hu} \mathrm{ID}^{3,6}$, Liping Mao ${ }^{1,2,3}$, Xiujin Ye ${ }^{1,2,3}$, Fang Liu $^{7^{\prime}}$, Zhenfang $\mathrm{Xi}^{8}$, Lihong Shou ${ }^{9}$, Caiyun $\mathrm{Fu}^{10}$, Hua Naranmandura (D) ${ }^{1,5^{\prime}}$, Jie Jin (iD) $1,2,3,4 \bowtie$ and Hong-Hu Zhu (iD) $1,2,3,4,11 \bowtie$

${ }^{1}$ Department of Hematology, The First Affiliated Hospital, Zhejiang University School of Medicine, Hangzhou, PR China. ${ }^{2}$ Zhejiang Provincial Key Lab of Hematopoietic Malignancy, Zhejiang University, Hangzhou, Zhejiang, PR China. ${ }^{3}$ Institute of Hematology, Zhejiang University, Hangzhou, Zhejiang, PR China. ${ }^{4}$ Zhejiang University Cancer Center, Hangzhou, Zhejiang, PR China. ${ }^{5}$ Department of Public Health, Zhejiang University School of Medicine, Hangzhou, PR China.

${ }^{6}$ Bone Marrow Transplantation Center, the First Affiliated Hospital, Zhejiang University School of Medicine, Hangzhou, PR China. ${ }^{7}$ Department of Hematology, Chengdu Military General Hospital, Chengdu, Sichuan, P.R. China. ${ }^{8}$ Department of Hematology, Linfen People's Hospital, Linfen, Shanxi, PR China. ${ }^{9}$ Department of Hematology, The Central Hospital of Huzhou City, Huzhou, People's Republic of China. ${ }^{10}$ College of Life Sciences and Medicine, Zhejiang Sci-Tech University, Hangzhou, China. ${ }^{11}$ Zhejiang Laboratory for Systems \& Precision Medicine, Zhejiang University Medical Center, Hangzhou, China. ${ }^{12}$ These authors contributed equally: Huafeng Wang, Chang Yang, Ting Shi, Yi Zhang, Jiejing Qian 凶email: narenman@zju.edu.cn; jiej0503@zju.edu.cn; zhuhhdoc@163.com

\section{REFERENCES}

1. Foa R, Bassan R, Vitale A, Elia L, Piciocchi A, Puzzolo $M C$, et al. DasatinibBlinatumomab for Ph-positive acute lymphoblastic leukemia in adults. $\mathrm{N}$ Engl J Med. 2020;383:1613-23.

2. Ravandi F. How I treat Philadelphia chromosome-positive acute lymphoblastic leukemia. Blood. 2019;133:130-6.

3. Cortes JE, Kim DW, Pinilla-Ibarz J, le Coutre P, Paquette R, Chuah C, et al. A phase 2 trial of ponatinib in Philadelphia chromosome-positive leukemias. N Engl J Med. 2013;369:1783-96.

4. Kim DY, Joo YD, Lim SN, Kim SD, Lee JH, Lee JH, et al. Nilotinib combined with multiagent chemotherapy for newly diagnosed Philadelphia-positive acute lymphoblastic leukemia. Blood. 2015;126:746-56.

5. Ting S, Mixue X, Lixia Z, Xueying L, Wanzhuo X, Xiujin Y. T315I mutation exerts a dismal prognosis on adult BCR-ABL1-positive acute lymphoblastic leukemia, and salvage therapy with ponatinib or CAR-T cell and bridging to allogeneic hematopoietic stem cell transplantation can improve clinical outcomes. Ann Hematol. 2020;99:829-34.

6. Scherr M, Elder A, Battmer K, Barzan D, Bomken S, Ricke-Hoch M, et al. Differential expression of miR-17 92 identifies BCL2 as a therapeutic target in BCR-ABLpositive B-lineage acute lymphoblastic leukemia. Leukemia. 2014;28:554-65.

7. Leonard JT, Rowley JS, Eide CA, Traer E, Hayes-Lattin B, Loriaux M, et al. Targeting $B C L-2$ and $A B L / L Y N$ in Philadelphia chromosome-positive acute lymphoblastic leukemia. Sci Transl Med. 2016;8:354ra114.

8. Scherr M, Kirchhoff H, Battmer K, Wohlan K, Lee CW, Ricke-Hoch M, et al. Optimized induction of mitochondrial apoptosis for chemotherapy-free treatment of BCR-ABL+acute lymphoblastic leukemia. Leukemia. 2019;33:1313-23.

9. Brown PA, Shah B, Advani A, Aoun P, Boyer MW, Burke PW, et al. Acute Lymphoblastic Leukemia, Version 2.2021, NCCN Clinical Practice Guidelines in Oncology. J Natl Compr Canc Netw. 2021;19:1079-109. 
10. Shah NP, Skaggs BJ, Branford S, Hughes TP, Nicoll JM, Paquette RL, et al. Sequential $A B L$ kinase inhibitor therapy selects for compound drug-resistant BCR-ABL mutations with altered oncogenic potency. J Clin Invest. 2007;117:2562-9.

11. Couturier MA, Thomas X, Raffoux E, Huguet F, Berthon C, Simand C, et al. Blinatumomab + ponatinib for relapsed/refractory Philadelphia chromosomepositive acute lymphoblastic leukemia in adults. Leuk Lymphoma. 2021;62:620-9.

12. Short NJ, Konopleva M, Kadia T, Kebriaei P, Daver N, Huang X, et al. An effective chemotherapy-free regimen of ponatinib plus venetoclax for relapsed/refractory Philadelphia chromosome-positive acute lymphoblastic leukemia. Am J Hematol. 2021;96:E229-E232.

\section{ACKNOWLEDGEMENTS}

This work was supported by grants (2020R01006 and 2019R01001 to Dr. Zhu) from the Leading Innovative and Entrepreneur Team Introduction Program of Zhejiang, grants ( 81820108004 and 82170144 to Dr. Jin) from the National Natural Science Foundation of China, grant (2021C03123 to Dr. Jin) from Key Research and Development Program of Zhejiang and grant (81872942 to Dr. Naranmandura) from National Natural Science Foundation of China.

\section{AUTHOR CONTRIBUTIONS}

HFW designed the study, analyzed results, and wrote the paper; $C Y, T S, Y Z$, and JJQ, collected data and analyzed results; YGW collected data and performed bcr/abl kinase domain mutation; YXH, LPM, XJY, LF, ZFX, LHS, and CYF collected data and reviewed the paper; $\mathrm{HN}, \mathrm{J}$, and $\mathrm{HHZ}$ designed the study, analyzed results, wrote the paper, and provided administrative support.

\section{COMPETING INTERESTS}

The authors declare no competing interests.

\section{ADDITIONAL INFORMATION}

Supplementary information The online version contains supplementary material available at https://doi.org/10.1038/s41408-022-00621-9.

Correspondence and requests for materials should be addressed to Hua Naranmandura, Jie Jin or Hong-Hu Zhu.

Reprints and permission information is available at http://www.nature.com/reprints

Publisher's note Springer Nature remains neutral with regard to jurisdictional claims in published maps and institutional affiliations.

(i) Open Access This article is licensed under a Creative Commons By Attribution 4.0 International License, which permits use, sharing, adaptation, distribution and reproduction in any medium or format, as long as you give appropriate credit to the original author(s) and the source, provide a link to the Creative Commons license, and indicate if changes were made. The images or other third party material in this article are included in the article's Creative Commons license, unless indicated otherwise in a credit line to the material. If material is not included in the article's Creative Commons license and your intended use is not permitted by statutory regulation or exceeds the permitted use, you will need to obtain permission directly from the copyright holder. To view a copy of this license, visit http://creativecommons. org/licenses/by/4.0/.

(c) The Author(s) 2022 